\title{
New generalized pseudodistance and coincidence point theorem in a $b$-metric space
}

\section{Robert Plebaniak*}

\section{"Correspondence:}

robpleb@math.uni.lodz.pl

Department of Nonlinear Analysis,

Faculty of Mathematics and

Computer Science, University of

Łódź, Banacha 22, Łódź, 90-238,

Poland

\begin{abstract}
In this paper, in a b-metric space, we introduce the concept of $b$-generalized pseudodistances which are the extension of $b$-metric. Next, inspired by ideas of Singh and Prasad we define a new contractive condition with respect to this $b$-generalized pseudodistance, and the condition guaranteeing the existence of coincidence points for four mappings. The examples which illustrate the main result are given. The paper includes also the comparison of our result with those existing in literature.
\end{abstract}

MSC: 47H10; 54H25; 54E50; 54E35; 45M20

Keywords: $b$-metric spaces; $b$-generalized pseudodistances; coincidence points theorem

\section{Introduction}

The study of existence and unique problems by iterative approximation originates from the work of Banach [1] concerning contractive maps.

Theorem 1.1 Let $(X, d)$ be a complete metric space and let $T: X \rightarrow X$. If

(B) (Banach [1]) $\exists_{0 \leq \lambda<1} \forall_{x, y \in X}\{d(T(x), T(y)) \leq \lambda d(x, y)\}$,

then: (a) $T$ has a unique fixed point $w$ in $X$; and (b) $\forall_{w^{0} \in X}\left\{\lim _{m \rightarrow \infty} T^{[m]}\left(w^{0}\right)=w\right\}$.

The Banach [1] result was an important tool to solve the following equation:

$$
T(x)=x,
$$

where $T: X \rightarrow X$ and $x \in X$. If we replace the identity map on $X$ with some map $S: X \rightarrow X$ on the right-hand side of equation (1.1), then we obtain the following equation:

$$
T(x)=S(x) .
$$

Equation (1.2) is called a coincidence point equation and plays a very important role in many physical formulations. To solve equation (1.2), we can use the Jungck [2, 3] iterative procedure, i.e.,

$$
S\left(x_{n+1}\right)=T\left(x_{n}\right), \quad n=0,1,2, \ldots
$$

In 1998, Czerwik [4] introduced the following definition of a $b$-metric space.

○2013 Plebaniak; licensee Springer. This is an Open Access article distributed under the terms of the Creative Commons Attribution License (http://creativecommons.org/licenses/by/2.0), which permits unrestricted use, distribution, and reproduction in any medium, provided the original work is properly cited. 
Definition 1.1 Let $X$ be a nonempty subset and $s \geq 1$ be a given real number. A function $d: X \times X \rightarrow[0, \infty)$ is $b$-metric if the following three conditions are satisfied:

(d1) $\forall_{x, y \in X}\{d(x, y)=0 \Leftrightarrow x=y\}$;

(d2) $\forall_{x, y \in X}\{d(x, y)=d(y, x)\}$; and

(d3) $\forall_{x, y, z \in X}\{d(x, z) \leq s[d(x, y)+d(y, z)]\}$.

The pair $(X, d)$ is called a $b$-metric space (with constant $s \geq 1$ ). It is easy to see that each metric space is a $b$-metric space.

Recently, in 2009, Singh and Prasad [5] introduced and established the following interesting and important coincidence points theorem for four maps in $b$-metric space.

Theorem 1.2 Let $(X, d)$ be a b-metric space (with $s \geq 1$ ), where $d: X \times X \rightarrow[0, \infty)$ is continuous on $X^{2}, Y \subseteq X$, and let $A, B, S, T: Y \rightarrow X$ be such that $T(Y) \subseteq B(Y), A(Y) \subseteq S(Y)$ and the following condition holds: there exists $q \in(0,1)$ such that $q s<1$ and $\lambda s<1$ (where $\left.\lambda=\max \left\{q, \frac{q s}{2-q s}\right\}\right)$ and such that for all $x, y \in X$, we have

$$
\begin{gathered}
d(T(x), A(y)) \leq q \max \{d(S(x), B(y)), d(S(x), T(x)), d(B(y), A(y)), \\
[d(S(x), A(y))+d(B(y), T(x 7))] / 2\} .
\end{gathered}
$$

If one of the images $A(Y), B(Y), S(Y)$ or $T(Y)$ is a complete subspace of $X$, then:

(i) $T$ and $S$ have a coincidence point, i.e., there exists $v \in Y$ such that $S(v)=T(v)$;

(ii) $A$ and $B$ have a coincidence point, i.e., there exists $w \in Y$ such that $B(w)=A(w)$.

It is worth noticing that condition (1.3) is a generalization of the following conditions, which are known in literature:

$$
\exists_{q \in(0,1)} \forall_{x, y \in Y}\{d(T(x), T(y)) \leq q d(S(x), S(y))\}
$$

and

$$
\begin{gathered}
\exists_{q \in(0,1)} \forall_{x, y \in Y}\{d(T(x), T(y)) \leq q \max \{d(S(x), S(y)), d(S(x), T(x)), \\
d(S(y), T(y)),[d(S(x), T(y))+d(S(y), T(x))] / 2\}\} .
\end{gathered}
$$

In literature, the pair of maps $S: Y \rightarrow X, T: Y \rightarrow X$ satisfying (1.4) is called the Jungck contraction, and $q$ is called the Jungck constant. Condition (1.5) with $Y=X$ and $S=i d$ (the identity map on $X$ ) was considered by Rhoades [6].

On the other hand, the famous Banach's result has been given extensive applications in many fields of mathematics and applied mathematics and has been extended in many different directions. One of the courses was to replace metric $d$ by some more general maps. In the complete metric spaces $(X, d), w$-distances [7] and $\tau$-distances [8] have been found to have substantial applications in fixed point theory. Due to them, some generalizations of Banach contractions have been introduced. Many interesting extensions of Theorem 1.1 to $w$-distances and $\tau$-distances settings have been given and techniques based on these distances have been presented (see, for example, [8, 9]). It is worth noticing that $\tau$-distances generalize $w$-distances and metrics $d$. In 2006, Włodarczyk and Plebaniak [10] introduced the concepts of $\mathcal{J}$-families of generalized pseudodistances in uniform spaces which generalized distances of Tataru [11], $w$-distances of Kada et al. [7], $\tau$-distances of Suzuki [12, 
Section 2] and $\tau$-functions of Lin and $\mathrm{Du}$ [13] in metric spaces and distances of Vályi [14] in uniform spaces. The distance was researched in [15-17].

The main interest of this paper is the following.

Question 1.1 Do a new kind of asymmetric distances (which extend $b$-metric) on $b$-metric spaces and a new kind of completeness of $b$-metric spaces exist?

Question 1.2 Does a new kind of contractions of (1.3) type with respect to these new distances exist?

The answer is affirmative. In this paper, in a $b$-metric space, we introduce the concept of $b$-generalized pseudodistances which are the extension of $b$-metric. Next, inspired by the ideas of Singh and Prasad [5], we define a new contractive condition of (1.3) type with respect to this $b$-generalized pseudodistance, and the condition guaranteeing the existence of coincidence points for four mappings. The examples which illustrate the main result are given. The paper includes also the comparison of our result with those existing in literature.

\section{On generalized pseudodistance, $b$-generalized pseudodistance and admissible $\boldsymbol{b}$-generalized pseudodistance in $\boldsymbol{b}$-metric spaces}

In the rest of the paper, we assume that the $b$-metric $d: X \times X \rightarrow[0, \infty)$ is continuous on $X^{2}$. At the very beginning, in a $b$-metric space, we introduce the concept of $b$-generalized pseudodistance, which is an essential generalization of $b$-metric.

Definition 2.1 Let $X$ be a $b$-metric space (with constant $s \geq 1$ ). The map $J: X \times X \rightarrow$ $[0, \infty)$ is said to be a generalized pseudodistance on $X$ if the following two conditions hold:

(J1) $\forall_{x, y, z \in X}\{J(x, z) \leq J(x, y)+J(y, z)\}$; and

(J2) For any sequences $\left(x_{m}: m \in \mathbb{N}\right)$ and $\left(y_{m}: m \in \mathbb{N}\right)$ in $X$ such that

$$
\lim _{n \rightarrow \infty} \sup _{m>n} J\left(x_{n}, x_{m}\right)=0
$$

and

$$
\lim _{m \rightarrow \infty} J\left(x_{m}, y_{m}\right)=0,
$$

we have

$$
\lim _{m \rightarrow \infty} d\left(x_{m}, y_{m}\right)=0
$$

Definition 2.2 Let $X$ be a $b$-metric space (with $s \geq 1$ ). The map $J: X \times X \rightarrow[0, \infty)$ is called a $b$-generalized pseudodistance on $X$ if the conditions (J1') and (J2) hold, where

(J1') $\forall_{x, y, z \in X}\{J(x, y) \leq s[J(x, z)+J(z, y)]\}$. 
Now, we introduce the following denotation. Let $X$ be a $b$-metric space (with $s \geq 1$ ), and let $J: X \times X \rightarrow[0, \infty)$ be a $b$-generalized pseudodistance on $X$. Then

$$
\begin{aligned}
& X_{J}^{0}=\{x \in X:\{J(x, x)=0\}\} \text { and } \\
& X_{J}^{+}=\{x \in X:\{J(x, x)>0\}\} .
\end{aligned}
$$

Then, of course, $X=X_{J}^{0} \cup X_{J}^{+}$.

Remark 2.1 (A) If $(X, d)$ is a $b$-metric space (with $s \geq 1$ ), then the $b$-metric $d: X \times X \rightarrow$ $[0, \infty)$ is a $b$-generalized pseudodistance on $X$. However, there exists a $b$-generalized pseudodistance on $X$ which is not $b$-metric (for details, see Example 4.1).

(B) It is clear that if the map $J$ is a generalized pseudodistance on $X$, then $J$ is a $b$-generalized pseudodistance on $X$ (for $s=1$ ).

(C) From (J1') and (J2) it follows that if $x \neq y, x, y \in X$, then

$$
J(x, y)>0 \vee J(y, x)>0 .
$$

Indeed, if $J(x, y)=0$ and $J(y, x)=0$, then $J(x, x)=0$, since by $\left(J 1^{\prime}\right)$ we get $J(x, x) \leq s[J(x, y)+$ $J(y, x)]=s[0+0]=0$. Now, defining $\left(x_{m}=x: m \in \mathbb{N}\right)$ and $\left(y_{m}=y: m \in \mathbb{N}\right)$, we conclude that (2.1) and (2.2) hold. Consequently, by (J2), we get (2.3), which implies $d(x, y)=0$. However, since $x \neq y$, we have $d(x, y) \neq 0$, a contradiction.

Now, we apply a $b$-generalized pseudodistance to establish a new kind of completeness, which is an extension of natural sequential completeness.

Definition 2.3 Let $(X, d)$ be a $b$-metric space (with $s \geq 1$ ), and let the map $J: X \times X \rightarrow$ $[0, \infty)$ be a $b$-generalized pseudodistance on $X$. We call that $X$ is $J$-complete if for all sequence $\left(x_{m}: m \in \mathbb{N}\right)$ in $X$ such that

$$
\lim _{n \rightarrow \infty} \sup _{m>n} J\left(x_{n}, x_{m}\right)=0,
$$

there exists $x \in X$ such that

$$
\lim _{m \rightarrow \infty} J\left(x_{m}, x\right)=\lim _{m \rightarrow \infty} J\left(x, x_{m}\right)=0 .
$$

Remark 2.2 It is worth noticing that if $J=d$, then by $(\mathrm{d} 2)$ the definitions of $J$-completeness and completeness are identical.

Definition 2.4 Let $(X, d)$ be a $b$-metric space (with $s \geq 1$ ), and let the map $J: X \times X \rightarrow$ $[0, \infty)$ be a $b$-generalized pseudodistance on $X$. We call that the map $J$ is admissible if for all the sequences $\left(x_{m}: m \in \mathbb{N}\right)$ and $\left(y_{m}: m \in \mathbb{N}\right)$ such that: (i) condition (2.1) (for these sequences, i.e., $\lim _{n \rightarrow \infty} \sup _{m>n} J\left(x_{n}, x_{m}\right)=0, \lim _{n \rightarrow \infty} \sup _{m>n} J\left(y_{n}, y_{m}\right)=0$ ) holds; and (ii) $\lim _{m \rightarrow \infty} d\left(x_{m}, x\right)=\lim _{m \rightarrow \infty} d\left(y_{m}, y\right)=0$; the following property is true:

$$
\lim _{m \rightarrow \infty} J\left(x_{m}, y_{m}\right)=J(x, y)
$$


Remark 2.3 (A) It is clear that if $x \in X_{J}^{0}$, then by (2.4) for a constant sequence $\left(x_{m}=x\right.$ : $m \in \mathbb{N})$, we have that

$$
\lim _{n \rightarrow \infty} \sup _{m>n} J\left(x_{n}, x_{m}\right)=\lim _{n \rightarrow \infty} \sup _{m>n} J(x, x)=0 .
$$

(B) Let $x \in X_{J}^{0}$ be arbitrary and fixed, and let $\left(x_{m}=x: m \in \mathbb{N}\right)$. Then, of course, by (d2) we obtain $\lim _{m \rightarrow \infty} d\left(x_{m}, x\right)=0$. Next, from (A) and Definition 2.4 it follows that if a sequence $\left(y_{m}: m \in \mathbb{N}\right)$ satisfies the following conditions: (i) $\lim _{n \rightarrow \infty} \sup _{m>n} J\left(y_{n}, y_{m}\right)=0$; and (ii) $\lim _{m \rightarrow \infty} d\left(y_{m}, y\right)=0$, then $\lim _{m \rightarrow \infty} J\left(x_{m}, y_{m}\right)=J(x, y)$. Moreover, similarly we can obtain that $\lim _{m \rightarrow \infty} J\left(y_{m}, x_{m}\right)=J(y, x)$.

Remark 2.4 It is worth noticing that if $(X, d)$ is a $b$-metric space (with $s \geq 1$ ), then the $b$-metric $d: X \times X \rightarrow[0, \infty)$ is an admissible $b$-generalized pseudodistance on $X$.

Definition 2.5 Let $(X, d)$ be a $b$-metric space (with $s \geq 1$ ), $Y \subseteq X$. Let $T: Y \rightarrow X$ and $S: Y \rightarrow X$ be single-valued maps. A point $z \in Y$ is called a coincidence point of $T$ and $S$ if $T(z)=S(z)=u$ for some $u \in X$.

The main result of this paper is the following coincidence theorem.

Theorem 2.1 Let $(X, d)$ be a b-metric space (with $s \geq 1$ ), $Y \subseteq X$, and let the map $J: X \times$ $X \rightarrow[0, \infty)$ be an admissible b-generalized pseudodistance on $X$. Let $A, B, S, T: Y \rightarrow X$ be such that $T(Y) \subseteq B(Y), A(Y) \subseteq S(Y)$. Let $T(Y) \subseteq X_{J}^{0}$ and $A(Y) \subseteq X_{J}^{0}$, and assume that the following condition holds: there exists $q \in(0,1)$ such that $\lambda s<1$ (where $\left.\lambda=\max \left\{q, \frac{q s}{2-q s}\right\}\right)$ and such that for all $x, y \in Y$ we have

$$
\begin{aligned}
\max & \{J(T(x), A(y)), J(A(y), T(x))\} \\
\leq & q \max \{\min \{J(S(x), B(y)), J(B(y), S(x))\}, \\
& J(S(x), T(x)), J(B(y), A(y)),[J(S(x), A(y))+J(B(y), T(x))] / 2\} .
\end{aligned}
$$

If one of the images of $Y$ under the mapping $A, B, S$ or $T$ is a J-complete subspace of $X$, then:

(i) $T$ and $S$ have a coincidence point $v \in Y$;

(ii) $A$ and $B$ have a coincidence point $z \in X$.

Moreover, for each $w^{0} \in Y$, if we define the sequences $\left(w^{n}: n \in\{0\} \cup \mathbb{N}\right)$ and $\left(v^{n}: n \in\right.$ $\{0\} \cup \mathbb{N}$ ) such that for each $n \in \mathbb{N}$ we get

$$
v^{2 n-1}=B\left(w^{2 n-1}\right)=T\left(w^{2 n-2}\right) \quad \text { and } \quad v^{2 n}=S\left(w^{2 n}\right)=A\left(w^{2 n-1}\right)
$$

then the sequence $\left(v^{n}: n \in\{0\} \cup \mathbb{N}\right)$ is convergent to $u\left(i . e ., \lim _{n \rightarrow \infty} d\left(v^{n}, u\right)=0\right)$, where

$$
u=T(v)=S(v)=A(z)=B(z)
$$




\section{Proof of Theorem 2.1}

Before starting the proof of Theorem 1.2, we present a simple consequence of property (2.5) and prove an auxiliary lemma. First, we can see that (2.5) implies that

$$
\begin{gathered}
J(T(x), A(y)) \leq q \max \{\min \{J(S(x), B(y)), J(B(y), S(x))\}, J(S(x), T(x)), \\
J(B(y), A(y)),[J(S(x), A(y))+J(B(y), T(x))] / 2\}
\end{gathered}
$$

and

$$
\begin{gathered}
J(A(y), T(x)) \leq q \max \{\min \{J(S(x), B(y)), J(B(y), S(x))\}, J(S(x), T(x)), \\
J(B(y), A(y)),[J(S(x), A(y))+J(B(y), T(x))] / 2\} .
\end{gathered}
$$

Lemma 3.1 Let $w^{0} \in Y$ be arbitrary and fixed. Then, for the sequences $\left(w^{n}: n \in\{0\} \cup \mathbb{N}\right)$ and $\left(v^{n}: n \in\{0\} \cup \mathbb{N}\right)$ defined as follows:

$$
\forall_{n \in \mathbb{N}}\left\{v^{2 n-1}=B\left(w^{2 n-1}\right)=T\left(w^{2 n-2}\right) \wedge v^{2 n}=S\left(w^{2 n}\right)=A\left(w^{2 n-1}\right)\right\},
$$

we have

$$
\exists_{\lambda=\max \left\{q, \frac{q s}{2-q s}\right\}<1} \forall_{n \in \mathbb{N}}\left\{J\left(v^{n+1}, v^{n+2}\right) \leq \lambda J\left(v^{n}, v^{n+1}\right)\right\} .
$$

Proof For fixed $n \in \mathbb{N}$, by (3.3) and (3.1) (for $x=w^{2 n-2}$ and $y=w^{2 n-1}$ ), we obtain

$$
\begin{aligned}
J\left(v^{2 n-1}, v^{2 n}\right)= & J\left(T\left(w^{2 n-2}\right), A\left(w^{2 n-1}\right)\right) \\
\leq & q \max \left\{\min \left\{J\left(S\left(w^{2 n-2}\right), B\left(w^{2 n-1}\right)\right), J\left(B\left(w^{2 n-1}\right), S\left(w^{2 n-2}\right)\right)\right\},\right. \\
& J\left(S\left(w^{2 n-2}\right), T\left(w^{2 n-2}\right)\right), J\left(B\left(w^{2 n-1}\right), A\left(w^{2 n-1}\right)\right), \\
& {\left.\left[J\left(S\left(w^{2 n-2}\right), A\left(w^{2 n-1}\right)\right)+J\left(B\left(w^{2 n-1}\right), T\left(w^{2 n-2}\right)\right)\right] / 2\right\} } \\
= & q \max \left\{\min \left\{J\left(v^{2 n-2}, v^{2 n-1}\right), J\left(v^{2 n-1}, v^{2 n-2}\right)\right\}, J\left(v^{2 n-2}, v^{2 n-1}\right), J\left(v^{2 n-1}, v^{2 n}\right),\right. \\
& {\left.\left[J\left(v^{2 n-2}, v^{2 n}\right)+J\left(v^{2 n-1}, v^{2 n-1}\right)\right] / 2\right\} . }
\end{aligned}
$$

Now, since by (3.3), $v^{2 n-1}=T\left(w^{2 n-2}\right)$ and by assumption $T(Y) \subseteq X_{J}^{0}$, we have $v^{2 n-1} \in X_{J}^{0}$ (i.e., $\left.J\left(v^{2 n-1}, v^{2 n-1}\right)=0\right)$. Consequently, by (3.4) we get

$$
\begin{aligned}
J\left(v^{2 n-1}, v^{2 n}\right) \leq & q \max \left\{\min \left\{J\left(v^{2 n-2}, v^{2 n-1}\right), J\left(v^{2 n-1}, v^{2 n-2}\right)\right\},\right. \\
& \left.J\left(v^{2 n-2}, v^{2 n-1}\right), J\left(v^{2 n-1}, v^{2 n}\right),\left[J\left(v^{2 n-2}, v^{2 n}\right)+J\left(v^{2 n-1}, v^{2 n-1}\right)\right] / 2\right\} \\
= & q \max \left\{\min \left\{J\left(v^{2 n-2}, v^{2 n-1}\right), J\left(v^{2 n-1}, v^{2 n-2}\right)\right\}, J\left(v^{2 n-2}, v^{2 n-1}\right)\right. \\
& \left.J\left(v^{2 n-1}, v^{2 n}\right), J\left(v^{2 n-2}, v^{2 n}\right) / 2\right\} .
\end{aligned}
$$

Let us consider the following three cases.

Case 1. If

$$
\begin{aligned}
& \max \left\{\min \left\{J\left(v^{2 n-2}, v^{2 n-1}\right), J\left(v^{2 n-1}, v^{2 n-2}\right)\right\}, J\left(v^{2 n-2}, v^{2 n-1}\right), J\left(v^{2 n-1}, v^{2 n}\right), J\left(v^{2 n-2}, v^{2 n}\right) / 2\right\} \\
& \quad=\min \left\{J\left(v^{2 n-2}, v^{2 n-1}\right), J\left(v^{2 n-1}, v^{2 n-2}\right)\right\}
\end{aligned}
$$


or

$$
\begin{aligned}
& \max \left\{\min \left\{J\left(v^{2 n-2}, v^{2 n-1}\right), J\left(v^{2 n-1}, v^{2 n-2}\right)\right\}, J\left(v^{2 n-2}, v^{2 n-1}\right), J\left(v^{2 n-1}, v^{2 n}\right), J\left(v^{2 n-2}, v^{2 n}\right) / 2\right\} \\
& \quad=J\left(v^{2 n-2}, v^{2 n-1}\right)
\end{aligned}
$$

then, since $\min \left\{J\left(v^{2 n-2}, v^{2 n-1}\right), J\left(v^{2 n-1}, v^{2 n-2}\right)\right\} \leq J\left(v^{2 n-2}, v^{2 n-1}\right)$, in both situations, by (3.5) we obtain

$$
J\left(v^{2 n-1}, v^{2 n}\right) \leq q J\left(v^{2 n-2}, v^{2 n-1}\right)
$$

Case 2. If

$$
\begin{aligned}
& \max \left\{\min \left\{J\left(v^{2 n-2}, v^{2 n-1}\right), J\left(v^{2 n-1}, v^{2 n-2}\right)\right\}, J\left(v^{2 n-2}, v^{2 n-1}\right), J\left(v^{2 n-1}, v^{2 n}\right), J\left(v^{2 n-2}, v^{2 n}\right) / 2\right\} \\
& \quad=J\left(v^{2 n-1}, v^{2 n}\right)
\end{aligned}
$$

then by (3.5) we have

$$
J\left(v^{2 n-1}, v^{2 n}\right) \leq q J\left(v^{2 n-1}, v^{2 n}\right)<J\left(v^{2 n-1}, v^{2 n}\right),
$$

which gives

$$
J\left(v^{2 n-1}, v^{2 n}\right)=0 .
$$

Case 3. If

$$
\begin{aligned}
& \max \left\{\min \left\{J\left(v^{2 n-2}, v^{2 n-1}\right), J\left(v^{2 n-1}, v^{2 n-2}\right)\right\}, J\left(v^{2 n-2}, v^{2 n-1}\right), J\left(v^{2 n-1}, v^{2 n}\right), J\left(v^{2 n-2}, v^{2 n}\right) / 2\right\} \\
& \quad=J\left(v^{2 n-2}, v^{2 n}\right) / 2
\end{aligned}
$$

then by (3.5) and $\left(\mathrm{J1}^{\prime}\right)$ we get

$$
\begin{aligned}
J\left(v^{2 n-1}, v^{2 n}\right) & \leq q J\left(v^{2 n-2}, v^{2 n}\right) / 2 \\
& \leq \frac{\left.q s J\left(v^{2 n-2}, v^{2 n-1}\right)+J\left(v^{2 n-1}, v^{2 n}\right)\right]}{2} \\
& =\frac{q s J\left(v^{2 n-2}, v^{2 n-1}\right)+q s J\left(v^{2 n-1}, v^{2 n}\right)}{2} .
\end{aligned}
$$

Hence

$$
J\left(v^{2 n-1}, v^{2 n}\right)-\frac{q S J\left(v^{2 n-1}, v^{2 n}\right)}{2} \leq \frac{q S J\left(v^{2 n-2}, v^{2 n-1}\right)}{2},
$$

which gives

$$
\frac{2-q s}{2} J\left(v^{2 n-1}, v^{2 n}\right) \leq \frac{q s}{2} J\left(v^{2 n-2}, v^{2 n-1}\right) .
$$

In consequence, we obtain

$$
J\left(v^{2 n-1}, v^{2 n}\right) \leq \frac{q s}{2-q s} J\left(v^{2 n-2}, v^{2 n-1}\right) .
$$


Conditions (3.6)-(3.8) imply that

$$
J\left(v^{2 n-1}, v^{2 n}\right) \leq \max \left\{q, \frac{q s}{2-q s}\right\} J\left(v^{2 n-2}, v^{2 n-1}\right) .
$$

Similarly, for fixed $n \in \mathbb{N}$, by (3.3) and (3.2) (for $x=w^{2 n}$ and $y=w^{2 n-1}$ ), we obtain

$$
\begin{aligned}
J\left(v^{2 n}, v^{2 n+1}\right)= & J\left(A\left(w^{2 n-1}\right), T\left(w^{2 n}\right)\right) \\
\leq & q \max \left\{\min \left\{J\left(S\left(w^{2 n}\right), B\left(w^{2 n-1}\right)\right), J\left(B\left(w^{2 n-1}\right), S\left(w^{2 n}\right)\right)\right\},\right. \\
& J\left(S\left(w^{2 n}\right), T\left(w^{2 n}\right)\right), J\left(B\left(w^{2 n-1}\right), A\left(w^{2 n-1}\right)\right), \\
& {\left.\left[J\left(S\left(w^{2 n}\right), A\left(w^{2 n-1}\right)\right)+J\left(B\left(w^{2 n-1}\right), T\left(w^{2 n}\right)\right)\right] / 2\right\} } \\
= & q \max \left\{\min \left\{J\left(v^{2 n}, v^{2 n-1}\right), J\left(v^{2 n-1}, v^{2 n}\right)\right\}, J\left(v^{2 n}, v^{2 n+1}\right),\right. \\
& \left.J\left(v^{2 n-1}, v^{2 n}\right),\left[J\left(v^{2 n}, v^{2 n}\right)+J\left(v^{2 n-1}, v^{2 n+1}\right)\right] / 2\right\} .
\end{aligned}
$$

Now, since by (3.3), $v^{2 n}=A\left(w^{2 n-1}\right)$ and by assumption $A(Y) \subseteq X_{J}^{0}$, we have $v^{2 n} \in X_{J}^{0}$ (i.e., $\left.J\left(v^{2 n}, v^{2 n}\right)=0\right)$. Consequently, since

$$
\left[J\left(v^{2 n}, v^{2 n}\right), J\left(v^{2 n-1}, v^{2 n+1}\right)\right] / 2=J\left(v^{2 n-1}, v^{2 n+1}\right) / 2,
$$

by (3.10) we get

$$
\begin{gathered}
J\left(v^{2 n}, v^{2 n+1}\right) \leq \\
q \max \left\{\min \left\{J\left(v^{2 n}, v^{2 n-1}\right), J\left(v^{2 n-1}, v^{2 n}\right)\right\}, J\left(v^{2 n}, v^{2 n+1}\right),\right. \\
\left.J\left(v^{2 n-1}, v^{2 n}\right), J\left(v^{2 n-1}, v^{2 n+1}\right) / 2\right\} .
\end{gathered}
$$

We will consider the following three cases.

Case 1. If

$$
\begin{aligned}
& \max \left\{\min \left\{J\left(v^{2 n}, v^{2 n-1}\right), J\left(v^{2 n-1}, v^{2 n}\right)\right\}, J\left(v^{2 n}, v^{2 n+1}\right), J\left(v^{2 n-1}, v^{2 n}\right), J\left(v^{2 n-1}, v^{2 n+1}\right) / 2\right\} \\
& \quad=\min \left\{J\left(v^{2 n}, v^{2 n-1}\right), J\left(v^{2 n-1}, v^{2 n}\right)\right\}
\end{aligned}
$$

or

$$
\begin{aligned}
& \max \left\{\min \left\{J\left(v^{2 n}, v^{2 n-1}\right), J\left(v^{2 n-1}, v^{2 n}\right)\right\}, J\left(v^{2 n}, v^{2 n+1}\right), J\left(v^{2 n-1}, v^{2 n}\right), J\left(v^{2 n-1}, v^{2 n+1}\right) / 2\right\} \\
& \quad=J\left(v^{2 n-1}, v^{2 n}\right),
\end{aligned}
$$

then, since $\min \left\{J\left(v^{2 n}, v^{2 n-1}\right), J\left(v^{2 n-1}, v^{2 n}\right)\right\} \leq J\left(v^{2 n-1}, v^{2 n}\right)$, in both situations, by (3.11) we obtain

$$
J\left(v^{2 n}, v^{2 n+1}\right) \leq q J\left(v^{2 n-1}, v^{2 n}\right) .
$$

Case 2. If

$$
\begin{aligned}
& \max \left\{\min \left\{J\left(v^{2 n}, v^{2 n-1}\right), J\left(v^{2 n-1}, v^{2 n}\right)\right\}, J\left(v^{2 n}, v^{2 n+1}\right), J\left(v^{2 n-1}, v^{2 n}\right), J\left(v^{2 n-1}, v^{2 n+1}\right) / 2\right\} \\
& \quad=J\left(v^{2 n}, v^{2 n+1}\right),
\end{aligned}
$$


then by (3.11) we have

$$
J\left(v^{2 n}, v^{2 n+1}\right) \leq q J\left(v^{2 n}, v^{2 n+1}\right)<J\left(v^{2 n}, v^{2 n+1}\right),
$$

which gives

$$
J\left(v^{2 n}, v^{2 n+1}\right)=0 .
$$

Case 3. If

$$
\begin{aligned}
& \max \left\{\min \left\{J\left(v^{2 n}, v^{2 n-1}\right), J\left(v^{2 n-1}, v^{2 n}\right)\right\}, J\left(v^{2 n}, v^{2 n+1}\right), J\left(v^{2 n-1}, v^{2 n}\right), J\left(v^{2 n-1}, v^{2 n+1}\right) / 2\right\} \\
& \quad=J\left(v^{2 n-1}, v^{2 n+1}\right) / 2
\end{aligned}
$$

then, by (3.11) and $\left(J 1^{\prime}\right)$, we get

$$
\begin{aligned}
J\left(v^{2 n}, v^{2 n+1}\right) & \leq q J\left(v^{2 n-1}, v^{2 n+1}\right) / 2 \\
& \leq \frac{\left.q s J\left(v^{2 n-1}, v^{2 n}\right)+J\left(v^{2 n}, v^{2 n+1}\right)\right]}{2} \\
& =\frac{q s J\left(v^{2 n-1}, v^{2 n}\right)+q s J\left(v^{2 n}, v^{2 n+1}\right)}{2} .
\end{aligned}
$$

Hence

$$
J\left(v^{2 n}, v^{2 n+1}\right)-\frac{q S J\left(v^{2 n}, v^{2 n+1}\right)}{2} \leq \frac{q S J\left(v^{2 n-1}, v^{2 n}\right)}{2},
$$

which gives

$$
\frac{2-q S}{2} J\left(v^{2 n}, v^{2 n+1}\right) \leq \frac{q s}{2} J\left(v^{2 n-1}, v^{2 n}\right) .
$$

In consequence, we obtain

$$
J\left(v^{2 n}, v^{2 n+1}\right) \leq \frac{q s}{2-q s} J\left(v^{2 n-1}, v^{2 n}\right) .
$$

Conditions (3.12)-(3.14) imply that

$$
J\left(v^{2 n}, v^{2 n+1}\right) \leq \max \left\{q, \frac{q s}{2-q s}\right\} J\left(v^{2 n-1}, v^{2 n}\right) .
$$

Next, conditions (3.9) and (3.15) imply that

$$
\exists_{\lambda=\max \left\{q, \frac{q s}{2-q s}\right\}<1} \forall_{n \in \mathbb{N}}\left\{J\left(v^{n+1}, v^{n+2}\right) \leq \lambda J\left(v^{n}, v^{n+1}\right)\right\} .
$$

The proof of the lemma is now completed.

Now we can start the proof of the main theorem. 


\section{Proof of Theorem 2.1}

Step I. Let $w^{0} \in Y$ be arbitrary and fixed. Construct the sequences $\left(w^{n}: n \in\{0\} \cup \mathbb{N}\right)$ and $\left(v^{n}: n \in\{0\} \cup \mathbb{N}\right)$ as in (3.3), i.e., such that for each $n \in \mathbb{N}$ we get

$$
v^{2 n-1}=B\left(w^{2 n-1}\right)=T\left(w^{2 n-2}\right) \quad \text { and } \quad v^{2 n}=S\left(w^{2 n}\right)=A\left(w^{2 n-1}\right) .
$$

Then, by Lemma 3.1, we obtain

$$
\exists_{\lambda=\max \left\{q, \frac{q s}{2-q s}\right\}<1} \forall_{n \in \mathbb{N}}\left\{J\left(v^{n+1}, v^{n+2}\right) \leq \lambda J\left(v^{n}, v^{n+1}\right)\right\} .
$$

Step II. We show that the sequence $\left(v^{n}: n \in\{0\} \cup \mathbb{N}\right)$ satisfies the following equation:

$$
\lim _{n \rightarrow \infty} \sup _{m>n} J\left(v^{n}, v^{m}\right)=0
$$

Indeed, for arbitrary and fixed $n \in \mathbb{N}$ and all $m \in \mathbb{N}, m>n$, by $\left(J 1^{\prime}\right)$, we calculate

$$
\begin{aligned}
J\left(v^{n}, v^{m}\right) & \leq s J\left(v^{n}, v^{n+1}\right)+s J\left(v^{n+1}, v^{m}\right) \\
& \leq s J\left(v^{n}, v^{n+1}\right)+s\left(s J\left(v^{n+1}, v^{n+2}\right)+s J\left(v^{n+2}, v^{m}\right)\right) \\
& =s J\left(v^{n}, v^{n+1}\right)+s^{2} J\left(v^{n+1}, v^{n+2}\right)+s^{2}\left(s J\left(v^{n+2}, v^{n+3}\right)+s J\left(v^{n+3}, v^{m}\right)\right) \leq \cdots \\
& \leq \sum_{i=1}^{m-n} s^{i} J\left(v^{n+i-1}, v^{n+i}\right) .
\end{aligned}
$$

Hence, by (3.16), since $\lambda<1$, we obtain

$$
\begin{aligned}
J\left(v^{n}, v^{m}\right) & \leq \sum_{i=1}^{m-n} s^{i} J\left(v^{n+i-1}, v^{n+i}\right) \leq \sum_{i=1}^{m-n} s^{i}\left[\lambda^{n+i-1} J\left(v^{0}, v^{1}\right)\right] \\
& =\sum_{i=1}^{m-n} s^{i} \lambda^{n+i-1} J\left(v^{0}, v^{1}\right)=J\left(v^{0}, v^{1}\right) \sum_{i=1}^{m-n} s^{i} \lambda^{n+i-1} \\
& =J\left(v^{0}, v^{1}\right) \lambda^{n-1} \sum_{i=1}^{m-n}(s \lambda)^{i}=J\left(v^{0}, v^{1}\right) \lambda^{n-1} \sum_{i=1}^{m-n} \gamma^{i} \\
& <\left[J\left(v^{0}, v^{1}\right) \sum_{i=1}^{m-n}\left(\gamma^{i}\right)\right] \cdot \lambda^{n-1},
\end{aligned}
$$

where $\gamma=s \lambda<1$. Therefore, (3.18) we have

$$
\begin{aligned}
\sup _{m>n} J\left(v^{n}, v^{m}\right) & \leq \sum_{m>n} J\left(v^{n}, v^{m}\right) \leq\left[J\left(v^{0}, v^{1}\right) \sum_{m=n+1}^{\infty} \sum_{i=1}^{m-n}\left(\gamma^{i}\right)\right] \cdot \lambda^{n-1} \\
& =\left[\frac{\gamma}{1-\gamma} J\left(v^{0}, v^{1}\right)\right] \cdot \lambda^{n-1} .
\end{aligned}
$$

Since $\lambda<1$, thus, as $n \rightarrow \infty$ in (3.19), we obtain

$$
\lim _{n \rightarrow \infty} \sup _{m>n} J\left(v^{n}, v^{m}\right)=0 .
$$

Thus, condition (3.17) holds. 
Step III. Now we show that if $S(Y)$ is J-complete, then there exists a unique $u \in S(Y)$ such that $\lim _{n \rightarrow \infty} v^{2 n}=u$.

Indeed, let $S(Y)$ be $J$-complete. By (3.17) and Definition 2.3, there exists $u \in S(Y)$ such that

$$
\lim _{n \rightarrow \infty} J\left(v^{n}, u\right)=\lim _{n \rightarrow \infty} J\left(u, v^{n}\right)=0 .
$$

The facts that $\lim _{n \rightarrow \infty} v^{n}=u$ and the point $u$ is unique are proved together. Indeed, let us suppose that there exists $\widetilde{u} \in S(Y), \widetilde{u} \neq u$, such that

$$
\lim _{n \rightarrow \infty} J\left(v^{n}, \widetilde{u}\right)=\lim _{n \rightarrow \infty} J\left(\widetilde{u}, v^{n}\right)=0 .
$$

Then for sequences $\left(x_{n}=v^{n}: n \in \mathbb{N}\right),\left(y_{n}=u: n \in \mathbb{N}\right)$ and $\left(\widetilde{y_{n}}=\widetilde{u}: n \in \mathbb{N}\right)$, by (3.17), (3.20) and (3.21), we have, respectively,

$$
\begin{aligned}
& \lim _{n \rightarrow \infty} \sup _{m>n} J\left(x_{n}, x_{m}\right)=0, \\
& \lim _{n \rightarrow \infty} J\left(x_{n}, y_{n}\right)=0 \text { and } \\
& \lim _{n \rightarrow \infty} J\left(x_{n}, \tilde{y}_{n}\right)=0 .
\end{aligned}
$$

By (3.22) and (3.23), for sequences $\left(x_{n}: n \in \mathbb{N}\right)$ and $\left(y_{n}: n \in \mathbb{N}\right)$, properties (2.1) and (2.2) hold, and similarly by (3.22) and (3.24), for sequences $\left(x_{n}: n \in \mathbb{N}\right)$ and $\left(\tilde{y_{n}}: n \in \mathbb{N}\right)$, properties (2.1) and (2.2) also hold. Hence, by (J2) we obtain

$$
\lim _{n \rightarrow \infty} d\left(v^{n}, u\right)=\lim _{n \rightarrow \infty} d\left(x_{n}, y_{n}\right)=0
$$

and

$$
\lim _{n \rightarrow \infty} d\left(v^{n}, \widetilde{u}\right)=\lim _{n \rightarrow \infty} d\left(x_{n}, \widetilde{y_{n}}\right)=0 .
$$

Now, from (3.25), (3.26) and (d1)-(d3), since $\tilde{u} \neq u$, we have that

$$
\forall_{n \in \mathbb{N}}\left\{0<\eta=d(\widetilde{u}, u) \leq s\left[d\left(\widetilde{u}, v^{n}\right)+d\left(v^{n}, u\right)\right]=s d\left(v^{n}, \widetilde{u}\right)+s d\left(v^{n}, u\right)\right\}
$$

Finally, by (3.27), (3.26) and (3.25), we have $0<\eta=d(\widetilde{u}, u) \leq s \lim _{n \rightarrow \infty} d\left(v^{n}, \tilde{u}\right)+$ $s \lim _{n \rightarrow \infty} d\left(v^{n}, u\right)=0$. Absurd.

Consequently, (3.20) holds for a unique $u$, and (3.25) gives that $\lim _{n \rightarrow \infty} v^{n}=u$.

Moreover, by (3.20), using a similar argumentation, for the subsequences $\left(v^{2 n}: n \in \mathbb{N}\right)$ and $\left(v^{2 n+1}: n \in \mathbb{N}\right)$, we have

$$
\lim _{n \rightarrow \infty} J\left(v^{2 n}, u\right)=\lim _{n \rightarrow \infty} J\left(u, v^{2 n}\right)=0,
$$

and

$$
\lim _{n \rightarrow \infty} J\left(v^{2 n+1}, u\right)=\lim _{n \rightarrow \infty} J\left(u, v^{2 n+1}\right)=0 .
$$


For clarity of the rest of the proof, let $v=S^{-1}(u)$. Then $S(v)=u$.

Step IV. We can show that

$$
J(S(v), u)=J(u, S(v))=0 .
$$

Indeed, by $\left(J 1^{\prime}\right)$ we have $\forall_{n \in \mathbb{N}}\left\{0 \leq J(u, u) \leq s J\left(u, v^{n}\right)+s J\left(v^{n}, u\right)\right\}$. Hence, by (3.20), we get

$$
0 \leq J(u, u) \leq s \lim _{n \rightarrow \infty} J\left(u, v^{n}\right)+s \lim _{n \rightarrow \infty} J\left(v^{n}, u\right)=0 .
$$

Thus, $u \in X_{J}^{0}$, i.e.,

$$
J(u, u)=0 .
$$

Now, since $S(v)=u$, we obtain that (3.28) holds.

Step V. We can show that

$$
\max \{J(T(v), u), J(u, T(v))\} \leq q J(S(v), T(v)) .
$$

Indeed, from (2.5) and (3.3), for $x=v$ and $y=w^{2 n+1}$, we calculate

$$
\begin{aligned}
\forall_{n \in \mathbb{N}} & \left\{\max \left\{J\left(T(v), v^{2 n+2}\right), J\left(v^{2 n+2}, T(v)\right)\right\}\right. \\
\quad & =\max \left\{J\left(T(v), A\left(w^{2 n+1}\right)\right), J\left(A\left(w^{2 n+1}\right), T(v)\right)\right\} \\
& \leq q \max \left\{\min \left\{J\left(S(v), B\left(w^{2 n+1}\right)\right), J\left(B\left(w^{2 n+1}\right), S(v)\right)\right\}, J(S(v), T(v)),\right. \\
& \left.J\left(B\left(w^{2 n+1}\right), A\left(w^{2 n+1}\right)\right),\left[J\left(S(v), A\left(w^{2 n+1}\right)\right)+J\left(B\left(w^{2 n+1}\right), T(v)\right)\right] / 2\right\} \\
& =q \max \left\{\min \left\{J\left(S(v), v^{2 n+1}\right), J\left(v^{2 n+1}, S(v)\right)\right\}, J(S(v), T(v)),\right. \\
& \left.\left.J\left(v^{2 n+1}, v^{2 n+2}\right),\left[J\left(S(v), v^{2 n+2}\right)+J\left(v^{2 n+1}, T(v)\right)\right] / 2\right\}\right\} .
\end{aligned}
$$

Now, since: (a) $T(v) \in T(Y) \subset X_{J}^{0}$; (b) $S(v)=u \in X_{J}^{0}$; (c) for sequences $\left(x_{n}=v^{2 n+2}: n \in \mathbb{N}\right)$, $\left(y_{n}=v^{2 n+1}: n \in \mathbb{N}\right)$ by Step III, we have $\lim _{n \rightarrow \infty} \sup _{m>n} J\left(x_{n}, x_{m}\right)=0, \lim _{n \rightarrow \infty} \sup _{m>n} J\left(y_{n}\right.$, $\left.y_{m}\right)=0$; (d) $\lim _{n \rightarrow \infty} x_{n}=\lim _{n \rightarrow \infty} y_{n}=u$; thus using the fact that $J$ is admissible, by Remark 2.3, we have:

(i) $\lim _{n \rightarrow \infty} J\left(T(v), v^{2 n+2}\right)=\lim _{n \rightarrow \infty} J\left(T(v), x_{n}\right)=J(T(v), u)$;

(ii) $\lim _{n \rightarrow \infty} J\left(v^{2 n+2}, T(v)\right)=\lim _{n \rightarrow \infty} J\left(x_{n}, T(v)\right)=J(u, T(v))$;

(iii) $\lim _{n \rightarrow \infty} J\left(S(v), v^{2 n+1}\right)=\lim _{n \rightarrow \infty} J\left(S(v), y_{n}\right)=J(S(v), u)$;

(iv) $\lim _{n \rightarrow \infty} J\left(v^{2 n+1}, S(v)\right)=\lim _{n \rightarrow \infty} J\left(y_{n}, S(v)\right)=J(u, S(v))$;

(v) $\lim _{n \rightarrow \infty} J\left(v^{2 n+1}, v^{2 n+2}\right)=\lim _{n \rightarrow \infty} J\left(y_{n}, x_{n}\right)=J(u, u)=0$;

(vi) $\lim _{n \rightarrow \infty} J\left(S(v), v^{2 n+2}\right)=\lim _{n \rightarrow \infty} J\left(S(v), x_{n}\right)=J(S(v), u)$;

(vii) $\lim _{n \rightarrow \infty} J\left(v^{2 n+1}, T(v)\right)=\lim _{n \rightarrow \infty} J\left(y_{n}, T(v)\right)=J(u, T(v))$.

Hence, in the limit, (3.31), (3.28) and (3.29) give

$$
\begin{aligned}
& \max \{J(T(v), u), J(u, T(v))\} \\
& \quad \leq q \max \{\min \{J(S(v), u), J(u, S(v))\}, J(S(v), T(v)), J(u, u),[J(S(v), u)+J(u, T(v))] / 2\} \\
& \quad=q \max \{J(S(v), T(v)), J(S(v), T(v)) / 2\}=q J(S(v), T(v)),
\end{aligned}
$$

thus (3.30) holds. 
Step VI. We claim that

$$
J(S(v), T(v))=0 \wedge J(T(v), S(v))=0 .
$$

First, we can observe that

$$
J(S(v), T(v))=0 .
$$

Indeed, supposing this claim is not true, then

$$
J(S(v), T(v))>0 .
$$

By (3.34) and (3.30), since $S(v)=u$, we obtain that

$$
\begin{aligned}
0 & <J(S(v), T(v))=J(u, T(v)) \leq \max \{J(T(v), u), J(u, T(v))\} \\
& \leq q J(S(v), T(v))<J(S(v), T(v)) .
\end{aligned}
$$

Contradiction. Thus (3.33) holds. Now, by (3.30) and (3.33), we obtain

$$
0<J(T(v), S(v))=J(T(v), u) \leq \max \{J(T(v), u), J(u, T(v))\} \leq q J(S(v), T(v))=0 .
$$

Hence, by (3.33) we obtain that (3.32) holds.

Step VII. Now, we show that $S(v)=T(v)$.

Indeed, this is the consequence of (3.32) and Remark 2.1(C).

Step VIII. Now, we can show that

$$
J(B(z), A(z))=J(A(z), B(z))=0
$$

for some $z \in Y$.

Indeed, since $u=S(v)$, thus by Step VII, $u=T(v) \in T(Y) \subseteq B(Y)$, so there exists $z \in Y$ such that $u=B(z)$. Next, from (2.5) and (3.3) (for $x=w^{2 n}$ and $y=z$ ), we calculate

$$
\begin{aligned}
& \forall_{n \in \mathbb{N}}\left\{\max \left\{J\left(v^{2 n+1}, A(z)\right), J\left(A(z), v^{2 n+1}\right)\right\}\right. \\
& \quad=\max \left\{J\left(T\left(w^{2 n}\right), A(z)\right), J\left(A(z), T\left(w^{2 n}\right)\right)\right\} \\
& \quad \leq q \max \left\{\min \left\{J\left(S\left(w^{2 n}\right), B(z)\right), J\left(B(z), S\left(w^{2 n}\right)\right)\right\},\right. \\
& \quad J\left(S\left(w^{2 n}\right), T\left(w^{2 n}\right)\right), J(B(z), A(z)), \\
& \left.\quad\left[J\left(S\left(w^{2 n}\right), A(z)\right)+J\left(B(z), T\left(w^{2 n}\right)\right)\right] / 2\right\} \\
& \quad=q \max \left\{\min \left\{J\left(v^{2 n}, B(z)\right), J\left(B(z), v^{2 n}\right)\right\}, J\left(v^{2 n}, v^{2 n+1}\right),\right. \\
& \left.\left.\quad J(B(z), A(z)),\left[J\left(v^{2 n}, A(z)\right)+J\left(B(z), v^{2 n+1}\right)\right] / 2\right\}\right\} .
\end{aligned}
$$

Now, since: (a) $A(z) \in A(Y) \subset X_{J}^{0}$; (b) $B(z)=u \in X_{J}^{0}$; (c) for sequences $\left(x_{n}=v^{2 n}: n \in \mathbb{N}\right)$, $\left(y_{n}=v^{2 n+1}: n \in \mathbb{N}\right)$ by Step III, we have $\lim _{n \rightarrow \infty} \sup _{m>n} J\left(x_{n}, x_{m}\right)=0, \lim _{n \rightarrow \infty} \sup _{m>n} J\left(y_{n}\right.$, $\left.y_{m}\right)=0$; (d) $\lim _{n \rightarrow \infty} x_{n}=\lim _{n \rightarrow \infty} y_{n}=u$; thus using the fact that $J$ is admissible, by Remark 2.3, we have: 
(i) $\lim _{n \rightarrow \infty} J\left(v^{2 n+1}, A(z)\right)=\lim _{n \rightarrow \infty} J\left(y_{n}, A(z)\right)=J(u, A(z))$;

(ii) $\lim _{n \rightarrow \infty} J\left(A(z), v^{2 n+1}\right)=\lim _{n \rightarrow \infty} J\left(A(z), y_{n}\right)=J(A(z), u)$;

(iii) $\lim _{n \rightarrow \infty} J\left(v^{2 n}, B(z)\right)=\lim _{n \rightarrow \infty} J\left(x_{n}, B(z)\right)=J(u, B(z))$;

(iv) $\lim _{n \rightarrow \infty} J\left(B(z), v^{2 n}\right)=\lim _{n \rightarrow \infty} J\left(B(z), x_{n}\right)=J(B(z), u)$;

(v) $\lim _{n \rightarrow \infty} J\left(v^{2 n}, v^{2 n+1}\right)=\lim _{n \rightarrow \infty} J\left(x_{n}, y_{n}\right)=J(u, u)=0$;

(vi) $\lim _{n \rightarrow \infty} J\left(v^{2 n}, A(z)\right)=\lim _{n \rightarrow \infty} J\left(x_{n}, A(z)\right)=J(u, A(z))$;

(vii) $\lim _{n \rightarrow \infty} J\left(B(z), v^{2 n+1}\right)=\lim _{n \rightarrow \infty} J\left(B(z), y_{n}\right)=J(B(z), u)$.

Hence, in the limit, (3.36) gives

$$
\begin{aligned}
\max \{J(u, A(z)), J(A(z), u)\} \leq & q \max \{\min \{J(u, B(z)), J(B(z), u)\} \\
& J(u, u), J(B(z), A(z)),[J(u, A(z))+J(B(z), u)] / 2\},
\end{aligned}
$$

and consequently, since $u=B(z)$ and $u=S(v)$, by (3.37) and (3.28), we obtain that

$$
\begin{aligned}
\max & \{J(B(z), A(z)), J(A(z), B(z))\} \\
& \leq q \max \{J(u, u), J(u, u), J(B(z), A(z)),[J(u, A(z))+J(u, u)] / 2\} \\
& =q \max \{J(u, S(v)), J(u, S(v)), J(B(z), A(z)),[J(B(z), A(z))+J(u, S(v))] / 2\} \\
& =q \max \{J(B(z), A(z)),[J(B(z), A(z))] / 2\} \\
& =q J(B(z), A(z)) .
\end{aligned}
$$

Now, we can observe that

$$
J(B(z), A(z))=0 .
$$

Indeed, supposing this claim is not true, then

$$
J(B(z), A(z))>0 .
$$

By (3.40) and (3.38) we obtain that

$$
\begin{aligned}
0 & <J(B(z), A(z)) \leq \max \{J(B(z), A(z)), J(A(z), B(z))\} \\
& \leq q J(B(z), A(z))<J(B(z), A(z)) .
\end{aligned}
$$

Contradiction. Thus $J(B(z), A(z))=0$, i.e., (3.39) holds.

Moreover, by (3.38) we get $J(A(z), B(z)) \leq \max \{J(B(z), A(z)), J(A(z), B(z))\} \leq q J(B(z)$, $A(z))<J(B(z), A(z))$, which by (3.39) gives that

$$
J(A(z), B(z))=0 .
$$

In consequence of (3.41), (3.39), we get that (3.35) holds.

Step IX. Now, we show that $A(z)=B(z)$.

Indeed, this is the consequence of (3.35) and Remark 2.1(C).

Step X. Now, we see that $\lim _{n \rightarrow \infty} d\left(v^{n}, u\right)=0$. 
Indeed, since $u=S(v)$ and $u=B(z)$, thus by Steps VII and IX, we obtain that $u=S(v)=$ $T(v)=A(z)=B(z)$. Moreover, by (3.17) and (3.20) we know that for the sequence $\left(v^{n}: n \in\right.$ $\mathbb{N})$, conditions (2.1) and (2.2) respectively hold, thus using (J2) we obtain

$$
\lim _{n \rightarrow \infty} d\left(v^{n}, u\right)=0
$$

Step XI. If $A(Y), B(Y)$ or $T(Y)$ are J-complete, then the assertions (i) and (ii) hold.

Indeed, if $A(Y)$ is $J$-complete, then since $A(Y) \subset S(Y)$, the assertions (i) and (ii) are true. If $B(Y)$ or $T(Y)$ is $J$-complete, then an analogous argument as that in Steps I-IX yields (i) and (ii).

\section{Remarks, examples and comparison}

Now, we present some examples illustrating the concepts which have been introduced so far. We will show a fundamental difference between Theorem 1.2 and Theorem 2.1. At the very beginning, we give the following remark.

Remark 4.1 (A) We can observe that if $(X, d)$ is a $b$-metric space (with $s \geq 1$ ) and $J=d$, then Theorem 2.1 and Theorem 1.2 are identical. Indeed, if $J=d$, then:

(1) $b$-metric $d: X \times X \rightarrow[0, \infty)$ is a $b$-generalized pseudodistance on $X$ (see Remark 2.1(A));

(2) $b$-metric $d: X \times X \rightarrow[0, \infty)$ is an admissible $b$-generalized pseudodistance on $X$ (see Remark 2.4);

(3) from (d1) and (2.4) we have $X_{d}^{0}=X$, and consequently $T(Y) \subseteq X=X_{d}^{0}$ and $A(Y) \subseteq X=X_{d}^{0}$

(4) definition of $J$-completeness and usual completeness of images $Y$ under the mapping $A, B, S$ or $T$ are identical (see Remark 2.2);

(5) from symmetry of $d$ (the property (d2)), we have that

$$
\begin{aligned}
& \forall_{x, y \in Y}\{\max \{J(T(x), A(y)), J(A(y), T(x))\} \\
& \quad=\max \{d(T(x), A(y)), d(A(y), T(x))\} \\
& \quad=d(T(x), A(y))\},
\end{aligned}
$$

and, similarly,

$$
\begin{aligned}
\forall_{x, y \in Y} & \{\min \{J(S(x), B(y)), J(B(y), S(x))\} \\
\quad= & \min \{d(S(x), B(y)), d(B(y), S(x))\} \\
\quad= & d(S(x), B(y))\},
\end{aligned}
$$

so conditions (2.5) and (1.3) are, in this case, identical.

(B) Generally, Theorem 2.1 is the essential extension of Theorem 1.2 (for details, see Example 4.3).

Now we show that Theorem 2.1 is the essential generalization of Theorem 1.2. First, we present an example of a $b$-generalized pseudodistance. 
Example 4.1 Let $X$ be a $b$-metric space (with a constant $s \geq 1$ ) equipped in $b$-metric $d$ : $X \times X \rightarrow[0, \infty)$. Let the closed set $E \subset X$, containing at least two different points, be arbitrary and fixed. Let $c>0$ be such that $c>\delta(E)$, where $\delta(E)=\sup \{d(x, y): x, y \in X\}$ is arbitrary and fixed. Define the map $J: X \times X \rightarrow[0, \infty)$ as follows:

$$
J(x, y)= \begin{cases}d(x, y) & \text { if }\{x, y\} \cap E=\{x, y\} \\ c & \text { if }\{x, y\} \cap E \neq\{x, y\}\end{cases}
$$

(I) We show that the map J is a b-generalized pseudodistance on X.

Indeed, it is worth noticing that the condition ( $\left.\mathrm{J1}^{\prime}\right)$ does not hold only if some $x_{0}, y_{0}, z_{0} \in$ $X$ such that $J\left(x_{0}, z_{0}\right)>s\left[J\left(x_{0}, y_{0}\right)+J\left(y_{0}, z_{0}\right)\right]$ exist. This inequality is equivalent to $c>$ $s\left[d\left(x_{0}, y_{0}\right)+d\left(y_{0}, z_{0}\right)\right]$, where $J\left(x_{0}, z_{0}\right)=c, J\left(x_{0}, y_{0}\right)=d\left(x_{0}, y_{0}\right)$ and $J\left(y_{0}, z_{0}\right)=d\left(y_{0}, z_{0}\right)$. However, by (4.1): $J\left(x_{0}, z_{0}\right)=c$ shows that there exists $v \in\left\{x_{0}, z_{0}\right\}$ such that $v \notin E$; $J\left(x_{0}, y_{0}\right)=$ $d\left(x_{0}, y_{0}\right)$ gives $\left\{x_{0}, y_{0}\right\} \subset E$; $J\left(y_{0}, z_{0}\right)=d\left(y_{0}, z_{0}\right)$ gives $\left\{y_{0}, z_{0}\right\} \subset E$. This is impossible. Therefore, $\forall_{x, y, z \in X}\{J(x, y) \leq s[J(x, z)+J(z, y)]\}$, i.e., the condition (J1') holds.

Proving that (J2) holds, we assume that the sequences $\left(x_{m}: m \in \mathbb{N}\right)$ and $\left(y_{m}: m \in \mathbb{N}\right)$ in $X$ satisfy (2.1) and (2.2). Then, in particular, (2.2) yields

$$
\forall_{0<\varepsilon<c} \exists_{m_{0}=m_{0}(\varepsilon) \in \mathbb{N}} \forall_{m \geq m_{0}}\left\{J\left(x_{m}, y_{m}\right)<\varepsilon\right\} .
$$

By (4.2) and (4.1), since $\varepsilon<c$, we conclude that

$$
\forall_{m \geq m_{0}}\left\{E \cap\left\{x_{m}, y_{m}\right\}=\left\{x_{m}, y_{m}\right\}\right\} .
$$

From (4.3), (4.1) and (4.2), we get

$$
\forall_{0<\varepsilon<c} \exists_{m_{0} \in \mathbb{N}} \forall_{m \geq m_{0}}\left\{d\left(x_{m}, y_{m}\right)<\varepsilon\right\} .
$$

Therefore, the sequences $\left(x_{m}: m \in \mathbb{N}\right)$ and $\left(y_{m}: m \in \mathbb{N}\right)$ satisfy (2.3). Consequently, the property (J2) holds.

(II) We will show that $J$ is an admissible b-generalized pseudodistance.

Indeed, let the sequences $\left(x_{m}: m \in \mathbb{N}\right)$ and $\left(y_{m}: m \in \mathbb{N}\right)$, such that $x_{m} \rightarrow x$ and $y_{m} \rightarrow y$, $m \rightarrow \infty$ and

$$
\begin{aligned}
& \lim _{n \rightarrow \infty} \sup _{m>n} J\left(x_{n}, x_{m}\right)=0, \quad \text { and } \\
& \lim _{n \rightarrow \infty} \sup _{m>n} J\left(y_{n}, y_{m}\right)=0,
\end{aligned}
$$

be arbitrary and fixed. Then by (4.4), (4.5) and (4.1) we obtain that

$$
\exists_{m_{0} \in \mathbb{N}} \forall_{m \geq m_{0}}\left\{\left\{x_{m}, y_{m}\right\} \cap E=\left\{x_{m}, y_{m}\right\}\right\},
$$

and by (4.1) we obtain

$$
\forall_{m \geq m_{0}}\left\{J\left(x_{m}, y_{m}\right)=d\left(x_{m}, y_{m}\right)\right\} .
$$


Moreover, since the set $E$ is closed, and $x_{m} \rightarrow x, y_{m} \rightarrow y$ by $m \rightarrow \infty$, so we have that $\{x, y\} \cap E=\{x, y\}$ and, consequently, by (4.1) we have

$$
J(x, y)=d(x, y) .
$$

Finally, (4.6), (4.7) and continuity of $d$ give that

$$
\lim _{m \rightarrow \infty} J\left(x_{m}, y_{m}\right)=\lim _{m \rightarrow \infty} d\left(x_{m}, y_{m}\right)=d(x, y)=J(x, y) .
$$

In the following, we illustrate how to satisfy condition (2.5) of Theorem 2.1 by an elementary example.

Example 4.2 Let $X$ be a $b$-metric space (with a constant $s=2>1$ ) equipped in $b$-metric $d$ : $X \times X \rightarrow[0, \infty)$, where $X=[1,5]$ and $d(x, y)=|x-y|^{2}, x, y \in X$. Let the set $E=\{2,3,4,5\} \subset$ $X$ and $J: X^{2} \rightarrow[0, \infty)$ be defined by the formula

$$
J(x, y)=\left\{\begin{array}{ll}
d(x, y) & \text { if } E \cap\{x, y\}=\{x, y\}, \\
10 & \text { if } E \cap\{x, y\} \neq\{x, y\},
\end{array} \quad x, y \in X .\right.
$$

Of course, $\delta(E)=\sup \left\{|x-y|^{2}: x, y \in E\right\}=9<10$, thus by Example 4.1(I) the map $J$ is the $b$-generalized pseudodistance on $X$. Moreover, since $E$ is a closed set, so by Example 4.1(II) the map $J$ is admissible on $X$.

Let $Y=[1,2] \subset X$ and let $T, A, S, B: Y \rightarrow X$ be given by the formulas

$$
\begin{aligned}
& T(x)= \begin{cases}3 & \text { if } x=1, \\
5 & \text { if } x \in\left\{\frac{5}{4}, \frac{3}{2}, \frac{7}{4}, 2\right\}, \\
4 & \text { if } x \in Y \backslash\left\{1, \frac{5}{4}, \frac{3}{2}, \frac{7}{4}, 2\right\},\end{cases} \\
& A(x)=\left\{\begin{array}{ll}
5 & \text { if } x \in Y \backslash\left\{\frac{4}{3}\right\}, \\
4 & \text { if } x=\frac{4}{3},
\end{array}\right. \text { and } \\
& S(x)= \begin{cases}\frac{15}{4} & \text { for } x \in Y \backslash\left\{\frac{5}{4}, \frac{3}{2}, \frac{7}{4}\right\}, \\
5 & \text { for } x=\frac{7}{4}, \\
4 & \text { for } x=\frac{5}{4}, \\
3 & \text { for } x=\frac{3}{2},\end{cases} \\
& B(x)= \begin{cases}\frac{15}{4} & \text { for } x \in Y \backslash\left\{\frac{5}{4}, \frac{3}{2}, 2\right\}, \\
5 & \text { for } x=\frac{5}{4}, \\
4 & \text { for } x=\frac{3}{2}, \\
3 & \text { for } x=2 .\end{cases}
\end{aligned}
$$

First, we can immediately see that $T(Y)=\{3,4,5\} \subset\left\{3, \frac{15}{4}, 4,5\right\}=B(Y)$ and $A(Y)=$ $\{4,5\} \subset\left\{3,4, \frac{15}{4}, 5\right\}=S(Y)$.

Now, we will show that the maps $T, A, S, B: Y \rightarrow X$ satisfy condition (2.5) for $\left(q=\frac{1}{4}\right)$. Indeed, first we can observe that since $s=2$, we get $q s=\frac{1}{2}<1$ and $\lambda s=\max \left\{q, \frac{q s}{2-q s}\right\} s=$ $2 \max \left\{\frac{1}{4}, \frac{\frac{1}{2}}{2-\frac{1}{2}}\right\}=2 \max \left\{\frac{1}{4}, \frac{1}{3}\right\}=\frac{2}{3}<1$. Moreover, since $T(Y)=\{3,4,5\} \subset E$ and $A(Y)=$ $\{4,5\} \subset E$, by (4.8) we get $T(Y) \subset X_{J}^{0}, A(Y) \subset X_{J}^{0}$ and

$$
\forall_{x, y \in Y}\{\max \{J(T(x), A(y)), J(A(y), T(x))\}=d(T(x), A(y)) \leq 2\} .
$$


Now, let $x, y \in Y$ be arbitrary and fixed. We consider the following four cases.

Case 1. If $\{x, y\} \cap\left\{\frac{5}{4}, \frac{3}{2}, \frac{7}{4}, 2\right\}=\varnothing$, then $\{x, y\} \subset Y \backslash\left\{\frac{5}{4}, \frac{3}{2}, \frac{7}{4}, 2\right\}$, which by (4.11) and (4.12) gives $B(x)=B(y)=S(x)=S(y)=\frac{15}{4}$ and $\frac{15}{4} \notin E$. By (4.8), we get $J(S(x), B(y))=10$, and consequently, since $\{S(x), B(y)\} \cap E=\emptyset$, by (4.8) we have that $J(S(x), T(x))=J(B(y), A(y))=$ $J(S(x), A(y))=J(B(y), T(x))=10$, thus

$$
\begin{gathered}
\max \{\min \{J(S(x), B(y)), J(B(y), S(x))\}, J(S(x), T(x)), J(B(y), A(y)), \\
[J(S(x), A(y))+J(B(y), T(x))] / 2\}=J(S(x), B(y))=10 .
\end{gathered}
$$

In consequence, by (4.13) and (4.14) we calculate

$$
\begin{aligned}
\max & \{J(T(x), A(y)), J(A(y), T(x))\} \\
\leq & 2<\frac{10}{4}=\frac{1}{4} \cdot 10 \\
= & q \max \{\min \{J(S(x), B(y)), J(B(y), S(x))\}, \\
& J(S(x), T(x)), J(B(y), A(y)),[J(S(x), A(y))+J(B(y), T(x))] / 2\} .
\end{aligned}
$$

Case 2. If $\{x, y\} \cap\left\{\frac{5}{4}, \frac{3}{2}, \frac{7}{4}, 2\right\}=\{x\}$, then $\{y\} \subset Y \backslash\left\{\frac{5}{4}, \frac{3}{2}, \frac{7}{4}, 2\right\}$, which by (4.12) gives $B(y)=$ $\frac{15}{4}$ and $\frac{15}{4} \notin E$. By (4.8), we get $J(S(x), B(y))=J(B(y), S(x))=10$, and consequently, since all images $T(Y), A(Y), S(Y)$ and $B(Y)$ are subsets of $E \cup\left\{\frac{15}{4}\right\}$, we have that $\delta(l) \leq 10$, where $l \in\{T(Y), A(Y), S(Y), B(Y)\}$. Hence, we calculate

$$
\begin{gathered}
\max \{\min \{J(S(x), B(y)), J(B(y), S(x))\}, J(S(x), T(x)), J(B(y), A(y)), \\
[J(S(x), A(y))+J(B(y), T(x))] / 2\}=J(S(x), B(y))=10 .
\end{gathered}
$$

In consequence, by (4.13) and (4.16) we calculate

$$
\begin{aligned}
\max & \{J(T(x), A(y)), J(A(y), T(x))\} \\
\leq & 2<\frac{10}{4}=\frac{1}{4} \cdot 10 \\
= & q \max \{\min \{J(S(x), B(y)), J(B(y), S(x))\}, \\
& J(S(x), T(x)), J(B(y), A(y)),[J(S(x), A(y))+J(B(y), T(x))] / 2\} .
\end{aligned}
$$

Case 3. If $\{x, y\} \cap\left\{\frac{5}{4}, \frac{3}{2}, \frac{7}{4}, 2\right\}=\{y\}$, then $\{x\} \subset Y \backslash\left\{\frac{5}{4}, \frac{3}{2}, \frac{7}{4}, 2\right\}$, which by (4.11) gives $S(x)=\frac{15}{4}$ and $\frac{15}{4} \notin E$. By (4.8), we get $=J(B(y), S(x))=10$, and consequently, since all images $T(Y), A(Y), S(Y)$ and $B(Y)$ are subsets of $E \cup\left\{\frac{15}{4}\right\}$, we have that $\delta(l) \leq 10$, where $l \in\{T(Y), A(Y), S(Y), B(Y)\}$. Hence, we calculate

$$
\begin{aligned}
\max & \{\min \{J(S(x), B(y)), J(B(y), S(x))\}, J(S(x), T(x)), J(B(y), A(y)), \\
& {[J(S(x), A(y))+J(B(y), T(x))] / 2\} } \\
= & J(S(x), B(y))=10 .
\end{aligned}
$$


In consequence, by (4.13) and (4.18) we calculate

$$
\begin{aligned}
\max & \{J(T(x), A(y)), J(A(y), T(x))\} \\
\leq & 2<\frac{10}{4}=\frac{1}{4} \cdot 10 \\
= & q \max \{\min \{J(S(x), B(y)), J(B(y), S(x))\}, J(S(x), T(x)), J(B(y), A(y)), \\
& {[J(S(x), A(y))+J(B(y), T(x))] / 2\} . }
\end{aligned}
$$

Case 4. If $\{x, y\} \cap\left\{\frac{5}{4}, \frac{3}{2}, \frac{7}{4}, 2\right\}=\{x, y\}$, then (4.9) and (4.10) give $T(x)=T(y)=A(x)=A(y)=$ 5 and $5 \in E$. By $(4.8)$, we get $J(T(x), A(y))=J(A(y), T(x))=d(T(x), A(y))=d(5,5)=0$, and consequently,

$$
\begin{aligned}
\max & \{J(T(x), A(y)), J(A(y), T(x))\} \\
=0 \leq & q \max \{\min \{J(S(x), B(y)), J(B(y), S(x))\}, \\
& J(S(x), T(x)), J(B(y), A(y)),[J(S(x), A(y))+J(B(y), T(x))] / 2\} .
\end{aligned}
$$

Consequently, (4.15), (4.17), (4.19) and (4.20) give that condition (2.5) holds.

Finally, we can observe, that: $T(Y) \subset X_{J}^{0} ; A(Y) \subset X_{J}^{0} ; X_{J}^{0}=E$; and $E$ is a closed set. Concluding, by (4.8) and Definition 2.3, we have $A(Y)$ and $T(Y)$ are $J$-complete subsets of $X$. All assumptions of Theorem 2.1 are satisfied. The maps $T$ and $S$ have a coincidence point $z=\frac{7}{4} \in Y$ (i.e., $T\left(\frac{7}{4}\right)=5=S\left(\frac{7}{4}\right)$ ), which presents that the assertion (i) holds, and $B$ and $A$ have a coincidence point $w=\frac{5}{4} \in Y$ (i.e., $A\left(\frac{5}{4}\right)=5=B\left(\frac{5}{4}\right)$ ), which gives that the assertion (ii) holds.

The next example illustrates that Theorem 2.1 is an essential extension of Theorem 1.2.

Example 4.3 Let $X$ be a $b$-metric space (with constant $s=2>1$ ) equipped in $b$-metric $d: X \times X \rightarrow[0, \infty)$, where $X=[1,5]$ and $d(x, y)=|x-y|^{2}, x, y \in X$. Let $Y \subset X$, and $A, B, S, T$ : $Y \rightarrow X$ be such as in Example 4.2. We will show that condition (1.3) does not hold. Indeed, supposing that there exists $q \in(0,1)$ such that $q s<1, \lambda s=\max \left\{q, \frac{q s}{2-q s}\right\} s<1$ and such that for each $x, y \in X$, we have

$$
\begin{gathered}
d(T(x), A(y)) \leq q \max \{d(S(x), B(y)), d(S(x), T(x)), d(B(y), A(y)), \\
[d(S(x), A(y))+d(B(y), T(x))] / 2\} .
\end{gathered}
$$

Let $x_{0}=1$ and $y_{0}=\frac{4}{3}$. Then by (4.9)-(4.12) we get:

(i) $d\left(T\left(x_{0}\right), A\left(y_{0}\right)\right)=d\left(T(1), A\left(\frac{4}{3}\right)\right)=d(3,4)=1$;

(ii) $d\left(S\left(x_{0}\right), B\left(y_{0}\right)\right)=d\left(S(1), B\left(\frac{4}{3}\right)\right)=d\left(\frac{15}{4}, \frac{15}{4}\right)=0$;

(iii) $d\left(S\left(x_{0}\right), T\left(x_{0}\right)\right)=d(S(1), T(1))=d\left(\frac{15}{4}, 3\right)=\left(\frac{3}{4}\right)^{2}=\frac{9}{16}$;

(iv) $d\left(B\left(y_{0}\right), A\left(y_{0}\right)\right)=d\left(B\left(\frac{4}{3}\right), A\left(\frac{4}{3}\right)\right)=d\left(\frac{15}{4}, 4\right)=\left(\frac{1}{4}\right)^{2}=\frac{1}{16}$;

(v) $\left[d\left(S\left(x_{0}\right), A\left(y_{0}\right)\right)+d\left(B\left(y_{0}\right), T\left(x_{0}\right)\right)\right] / 2=\left[d\left(S(1), A\left(\frac{4}{3}\right)\right)+d\left(B\left(\frac{4}{3}\right), T(1)\right)\right] / 2=$ $\left[d\left(\frac{15}{4}, 4\right)+d\left(\frac{15}{4}, 3\right)\right] / 2=(1 / 16+9 / 16) / 2=5 / 16 ;$ and

(vi) $\max \left\{d\left(S\left(x_{0}\right), B\left(y_{0}\right)\right), d\left(S\left(x_{0}\right), T\left(x_{0}\right)\right), d\left(B\left(y_{0}\right), A\left(y_{0}\right)\right),\left[d\left(S\left(x_{0}\right), A\left(y_{0}\right)\right)+\right.\right.$ $\left.\left.d\left(B\left(y_{0}\right), T\left(x_{0}\right)\right)\right] / 2\right\}=\max \{0,1 / 16,5 / 16,9 / 16\}=9 / 16$. 
Now, since $q<1$, by (i), (4.21) and (vi) we have

$$
\begin{aligned}
1= & d\left(T\left(x_{0}\right), A\left(y_{0}\right)\right) \\
\leq & q \max \left\{d\left(S\left(x_{0}\right), B\left(y_{0}\right)\right), d\left(S\left(x_{0}\right), T\left(x_{0}\right)\right),\right. \\
& \left.d\left(B\left(y_{0}\right), A\left(y_{0}\right)\right),\left[d\left(S\left(x_{0}\right), A\left(y_{0}\right)\right)+d\left(B\left(y_{0}\right), T\left(x_{0}\right)\right)\right] / 2\right\} \\
< & \max \left\{d\left(S\left(x_{0}\right), B\left(y_{0}\right)\right), d\left(S\left(x_{0}\right), T\left(x_{0}\right)\right), d\left(B\left(y_{0}\right), A\left(y_{0}\right)\right),\right. \\
& {\left.\left[d\left(S\left(x_{0}\right), A\left(y_{0}\right)\right)+d\left(B\left(y_{0}\right), T\left(x_{0}\right)\right)\right] / 2\right\}=\frac{9}{16}, }
\end{aligned}
$$

which is absurd. This shows that condition (1.3) does not hold, so the main assumption of Theorem 1.2 is not true.

Remark 4.2 Examples 4.2 and 4.3 show that there exist the maps and $b$-metrics such that we cannot use Theorem 1.2, but we can use Theorem 2.1.

\section{Competing interests}

The author declares that they have no competing interests.

Received: 17 June 2013 Accepted: 18 September 2013 Published: 08 Nov 2013

\section{References}

1. Banach, S: Sur les opérations dans les ensembles abstraits et leurs applications aux équations intégrales. Fundam. Math. 3, 133-181 (1922)

2. Jungck, G: Commuting mappings and fixed points. Am. Math. Mon. 83, 261-263 (1976)

3. Jungck, G: Common fixed points for commuting and compatible maps on compacta. Proc. Am. Math. Soc. 103, 977-983 (1988)

4. Czerwik, S: Nonlinear set-valued contraction mappings in b-metric spaces. Atti Semin. Mat. Fis. Univ. Modena 46(2), 263-276 (1998)

5. Singh, SL, Prasad, B: Some coincidence theorems and stability of iterative procedures. Comput. Math. Appl. 55, 2512-2520 (2008)

6. Rhoades, BE: A comparison of various definitions of contractive mappings. Trans. Am. Math. Soc. 226, 257-290 (1977)

7. Kada, O, Suzuki, T, Takahashi, W: Nonconvex minimization theorems and fixed point theorems in complete metric spaces. Math. Jpn. 44, 381-391 (1996)

8. Suzuki, T: Generalized distance and existence theorems in complete metric spaces. J. Math. Anal. Appl. 253, 440-458 (2001)

9. Suzuki, T: Several fixed point theorems concerning $\tau$-distance. Fixed Point Theory Appl. 2004, 195-209 (2004)

10. Włodarczyk, K, Plebaniak, R: Maximality principle and general results of Ekeland and Caristi types without lower semicontinuity assumptions in cone uniform spaces with generalized pseudodistances. Fixed Point Theory Appl. 2010, Article ID 175453 (2010)

11. Tataru, D: Viscosity solutions of Hamilton-Jacobi equations with unbounded nonlinear terms. J. Math. Anal. Appl. 163 345-392 (1992)

12. Suzuki, T: Generalized distance and existence theorems in complete metric spaces. J. Math. Anal. Appl. 253, 440-458 (2001)

13. Lin, L-J, Du, W-S: Ekeland's variational principle, minimax theorems and existence of nonconvex equilibria in complete metric spaces. J. Math. Anal. Appl. 323, 360-370 (2006)

14. Vályi, I: A general maximality principle and a fixed point theorem in uniform spaces. Period. Math. Hung. 16, 127-134 (1985)

15. Włodarczyk, K, Plebaniak, R: Periodic point, endpoint, and convergence theorems for dissipative set-valued dynamic systems with generalized pseudodistances in cone uniform and uniform spaces. Fixed Point Theory Appl. 2010 Article ID 864536 (2010)

16. Włodarczyk, K, Plebaniak, R, Doliński, M: Cone uniform, cone locally convex and cone metric spaces, endpoints, set-valued dynamic systems and quasi-asymptotic contractions. Nonlinear Anal. 71, 5022-5031 (2009)

17. Włodarczyk, K, Plebaniak, R, Obczyński, C: Convergence theorems, best approximation and best proximity for set-valued dynamic systems of relatively quasi-asymptotic contractions in cone uniform spaces. Nonlinear Anal. 72 794-805 (2010)

10.1186/1687-1812-2013-270

Cite this article as: Plebaniak: New generalized pseudodistance and coincidence point theorem in a $b$-metric space. Fixed Point Theory and Applications 2013, 2013:270 